\title{
Cavity-aided magnetic resonance microscopy of atomic transport in optical lattices
}

\author{
Nathan Brahms ${ }^{1 \star}$, Thomas P. Purdy ${ }^{1 \dagger}$, Daniel W. C. Brooks ${ }^{1}$, Thierry Botter ${ }^{1}$ \\ and Dan M. Stamper-Kurn ${ }^{1,2}$
}

\begin{abstract}
Ultracold atoms are emerging as an important platform for precision sensing and measurement, quantum information science, and simulations of condensed-matter phenomena. Microscopic imaging is a powerful tool for measuring cold-atom systems, enabling the readout of ultracold atomic simulators ${ }^{1,2}$ and registers ${ }^{3}$, the characterization of inhomogeneous environments ${ }^{4}$, and the determination of spatially varying thermodynamic quantities ${ }^{5-8}$. Cold-atom microscopy has recently been demonstrated with imaging resolution sufficient to detect and address single $\mathrm{e}^{9}$ or multiple $\mathrm{e}^{10}$ atoms at individual optical-lattice sites with lattice spacings of micrometres ${ }^{11,12}$ and below ${ }^{1,2,13,14}$. However, those methods, which rely either on the fluorescence ${ }^{1,2,9,11-13}$ or ionization ${ }^{10,14}$ of atoms, destroy the quantum states being measured and have limited dynamic range. Here we demonstrate magnetic resonance imaging of atomic gases in optical lattices, obtained by dispersively coupling atoms to a high-finesse optical cavity. We achieve statesensitive, single-lattice-site images with high dynamic range. We also apply this technique to measure the non-equilibrium transport dynamics of the gas.
\end{abstract}

The sensitivity of optical cavities has been used to make nondestructive ${ }^{15}$, state-sensitive ${ }^{16}$, and high-dynamic-range ${ }^{17}$ measurements of atomic gases, whereas magnetic resonance has been used to address the spin states of single atoms in single opticallattice $\operatorname{sites}^{9,18}$. In this experiment we use light in a high-finesse optical cavity, together with radio-frequency (RF) radiation and an inhomogeneous magnetic field, to perform real-time magnetic resonance imaging (MRI) of atomic spins in an optical lattice. We obtain a spatial resolution of $150 \mathrm{~nm}$, far below the $425 \mathrm{~nm}$ spacing of the lattice sites. We obtain a number-counting sensitivity of 10 for up to 1,000 atoms in each site or 2.5 for up to 70 atoms, both well below the level of Poissonian atom-number fluctuations. Furthermore, the MRI is minimally destructive of the atoms' internal states, allowing the single-site observation of spatially dependent spin dynamics. We use this technique to measure the transport dynamics of an initially localized gas via resonant quantum tunnelling between lattice sites, as the atoms undergo first ballistic and then interaction-inhibited transport. We also demonstrate the ability to address the spins of large numbers of atoms at selected lattice sites, enabling new studies of magnetism, transport, cavity spin optodynamics ${ }^{19}$, and cavity optomechanics ${ }^{20-22}$.

In contrast to traditional MRI, which measures transverse magnetization at the Larmor precession frequency, here we use the cavity to perform a quantum non-demolition measurement of the static longitudinal magnetization ${ }^{23}$ by applying a magnetic bias field along the cavity axis $\hat{k}$ (Fig. 1). To resolve the atoms spatially we apply a strong field gradient $B^{\prime}$ along the dimension

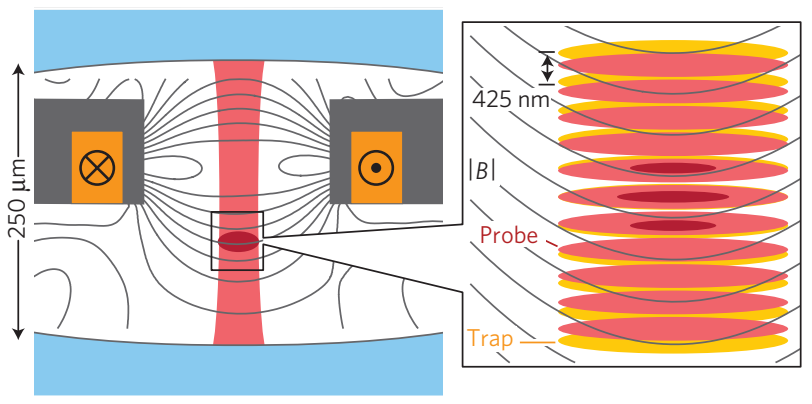

Figure 1 | An ensemble of ${ }^{87} \mathrm{Rb}$ atoms is optically trapped within a vertically oriented high-finesse Fabry-Perot cavity. Copper wires (orange, with current direction indicated) embedded within a $100-\mu m$-thick silicon substrate (grey), together with an external bias coil, produce both a strong vertical magnetic field gradient $(|B|$ contours shown) and a vertical bias field near the atoms. Inset: Atoms (red) are trapped at the antinodes of a standing-wave optical lattice (yellow) with $425 \mathrm{~nm}$ lattice spacing. Circularly polarized cavity probe light (pink), detuned several gigahertz from the $D_{2}$ line, acquires a dispersive phase shift that is sensitive to the atom spin projection along the cavity axis.

to be imaged. For the data presented here we image along the cavity axis, although any axis can be imaged by applying the appropriate gradient. We note especially that $3 \mathrm{D}$ images can be taken by imaging along a suitable number of axes and applying tomographic reconstruction ${ }^{24}$.

For a sufficiently large detuning $\Delta_{\text {ca }}$ between the cavity resonance frequency and the atomic optical resonance, the absorption of cavity light by the atoms is negligible, and the light-atom interaction may be described by a real-valued index of refraction. This index causes the cavity resonance frequency $\omega_{c}$ to be shifted from its empty-cavity value $\omega_{0}$. Because the atoms are circularly birefringent, the resonance frequency of circularly polarized light is shifted by an amount $\Delta_{N}$ that depends on the atom density $\rho(\mathbf{r})$ and the density $\mathbf{s}(\mathbf{r})$ of the dimensionless atomic spin (see the Supplementary Information):

$$
\Delta_{N} \equiv \omega_{\mathrm{c}}-\omega_{0}=\int g(\mathbf{r})[\rho(\mathbf{r}) \pm C \mathbf{s}(\mathbf{r}) \cdot \hat{k}] \mathrm{d}^{3} \mathbf{r}
$$

Here $g(\mathbf{r})$ is the scalar dispersive atom-cavity coupling parameter at position $\mathbf{r}$, taking into account the spatially varying intensity of the cavity mode. The \pm corresponds to $\sigma^{ \pm}$light polarizations, and $C$ is derived from a sum over probability amplitudes and relative detunings from the atomic excited hyperfine states. For

${ }^{1}$ Department of Physics, University of California, Berkeley, California 94720, USA, ${ }^{2}$ Materials Sciences Division, Lawrence Berkeley National Laboratory, Berkeley, California 94720, USA. †Present address: JILA, University of Colorado, Boulder, Colorado 80309, USA. *e-mail: nbrahms@berkeley.edu. 
a
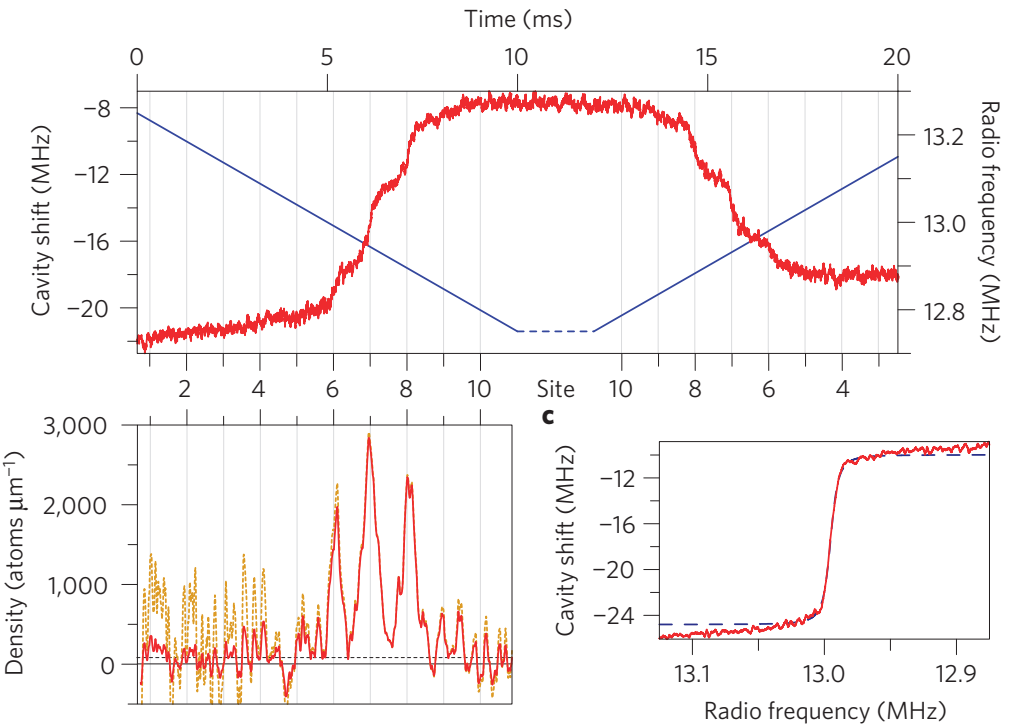

Figure 2 Single-shot MRI of atoms in an optical lattice. For this image, $\Delta_{\mathrm{ca}} / 2 \pi=-14 \mathrm{GHz}$ with $1 \times 10^{7}$ photons s $\mathrm{s}^{-1}$ exiting the cavity, and 1,800 atoms initially in the $\left|F, m_{F}\right\rangle=|2,2\rangle$ state. a, The shift $\Delta_{N} \equiv \omega_{\mathrm{C}}-\omega_{0}$ of the cavity from its empty resonance frequency (red line, left axis), showing steps as the RF (blue line, right axis) is chirped from high to low. As the RF is swept back, the detuning recovers its initial value with $85 \%$ each-way fidelity. $\mathbf{b}$, Atomic density as calculated from the time derivative of $\Delta_{N}$, both uncorrected (red solid line) and corrected (yellow dotted line) for spatially varying sensitivity (probe and trap antinodes are overlapped at site 7). The peak widths ( $200 \mathrm{~nm}$ FWHM) are given by the convolution of the imaging resolution (150 nm), the atom-distribution width $(100 \mathrm{~nm})$, and a low-pass analysis filter $(90 \mathrm{~nm})$. The image has an offset of 80 atom $\mu \mathrm{m}^{-1}$ due to deterministic atom loss (dotted line).c. The imaging resolution is proportional to the ratio of the $14 \mathrm{kHz}$ magnetic resonance width to the $114 \mathrm{kHz}_{\mu} \mathrm{m}^{-1}$ gradient of the magnetic resonance frequency. The magnetic resonance width is measured by sweeping over resonance in a uniform bias field (red solid line) and fitting to adiabatic-passage theory (blue dashed line).

${ }^{87} \mathrm{Rb}$ atoms with hyperfine spin $F=2$ and probe light detuned by several gigahertz from the $\mathrm{D}_{2}$ atomic resonance, $C \simeq 1 / 4$.

We invert the local magnetization by means of adiabatic passage using a chirped RF field. We use a linear chirp, with detuning from the magnetic resonance at position $z$ given at time $t$ by $\delta=\dot{\omega}_{\mathrm{rf}} t-\mu B^{\prime} z / \hbar$, where $\mu / \hbar$ is the atomic gyromagnetic ratio. The radially integrated density $s_{k}(z)$ of the dimensionless atomic spin can be extracted from the time derivative of the cavity frequency. For narrow magnetic resonance, where the spins at $z$ flip completely as the RF is swept from just below to just above resonance, the spin density is:

$$
s_{k}(z)=\left.\mp \frac{1}{2 C \bar{g}(z)} \frac{\mu B^{\prime}}{\hbar \dot{\omega}_{\mathrm{rf}}} \frac{\mathrm{d} \omega_{\mathrm{c}}}{\mathrm{d} t}\right|_{t=\mu B^{\prime} z / \hbar \dot{\omega}_{\mathrm{rf}}}
$$

Here $\bar{g}$ is the density-weighted radial average of the cavity coupling at $z$. We neglect the small radial variation of the magnetic field across the gas. This formula is easily refined to account for the finite frequency width of the magnetic resonance (see the Supplementary Information).

Experiments are conducted using a Fabry-Perot optical cavity integrated onto a microfabricated atom-chip device ${ }^{22}$. The chip is used for sample preparation, delivering atomic gases of up to 5,000 atoms, spin polarized in the $\left|F, m_{\mathrm{F}}\right\rangle=|2,2\rangle$ hyperfine state, at temperatures of $1-3 \mu \mathrm{K}$ into the optical lattice. Chip wires are then used to apply strong magnetic field gradients, allowing us to resolve and address individual lattice sites.

The optical cavity is driven with two different wavelengths of light. Light at a wavelength of $850 \mathrm{~nm}$ establishes a far-offresonant optical lattice potential. A cavity probe beam, detuned $14-17 \mathrm{GHz}$ to the red of the $\mathrm{D}_{2}$ atomic resonance at a wavelength of $780 \mathrm{~nm}$, measures the number of atoms and the spin densities as described above. The atoms are loaded into a few (between 2 and 5) adjacent lattice sites, centred on a site that overlaps with an antinode of the probe field.
To measure the cavity shift we detect the photon flux of $\sigma^{+}$polarized probe light transmitted through the cavity. A feedback loop tunes the probe frequency $\omega_{\mathrm{p}}$, maintaining the flux at a constant value $\bar{\gamma}$ equal to a fixed fraction of the incident photon flux. The probe frequency is thus locked at a fixed detuning $\Delta_{\mathrm{pc}}$ from cavity resonance, the frequency of which is determined by $\omega_{\mathrm{c}}=\omega_{\mathrm{p}}-\Delta_{\mathrm{pc}}$. Variations in the cavity resonance frequency faster than the $20 \mathrm{kHz}$ feedback bandwidth can be assessed by using the residual deviation of the instantaneous photon flux $\gamma$ from $\bar{\gamma}$.

Figure 2 shows a typical single-shot image of a spin-polarized atomic gas taken with a linear RF chirp. As the RF is swept through the resonance of each lattice site, atoms are flipped from the $|2,2\rangle$ to the $|2,-2\rangle$ state, causing a jump in the cavity resonance frequency. The atom density is obtained by calculating $s_{k}(z) / 2$ using equation (1). We account for the thermal radial and axial distribution of atoms within each lattice site in determining $\bar{g}(z)$. We also account for the difference between the trap and probe light wavelengths, although this latter correction is minor for lattice sites near the common antinode of the two cavity modes. Images may also be averaged (see Fig. 4), although shot-to-shot variations in the bias field cause broadening of the averaged images. The flatness of the slope of the cavity shift between spin flips verifies our ability to address single lattice sites (see the Supplementary Information).

The spatial resolution with which we can image the spins is $150 \mathrm{~nm}$ (full width at half-maximum). The resolution is proportional to the ratio of the magnetic resonance width $(14 \mathrm{kHz}$, see Fig. 2c) to the gradient of the magnetic resonance frequency $\left(114 \mathrm{kHz}_{\mu \mathrm{m}}^{-1}\right)$. The minimum resolution is limited by two considerations. First, the Rabi frequency must be sufficiently high that the spins are inverted adiabatically. Second, the maximum magnetic field gradient is limited by transverse field curvatures, which expel atoms from the optical trap (see the Supplementary Information).

The atomic magnetization is largely preserved during the imaging process and can be recovered by reversing the RF chirp, as shown in Fig. 2. The magnetization is slightly diminished as a 


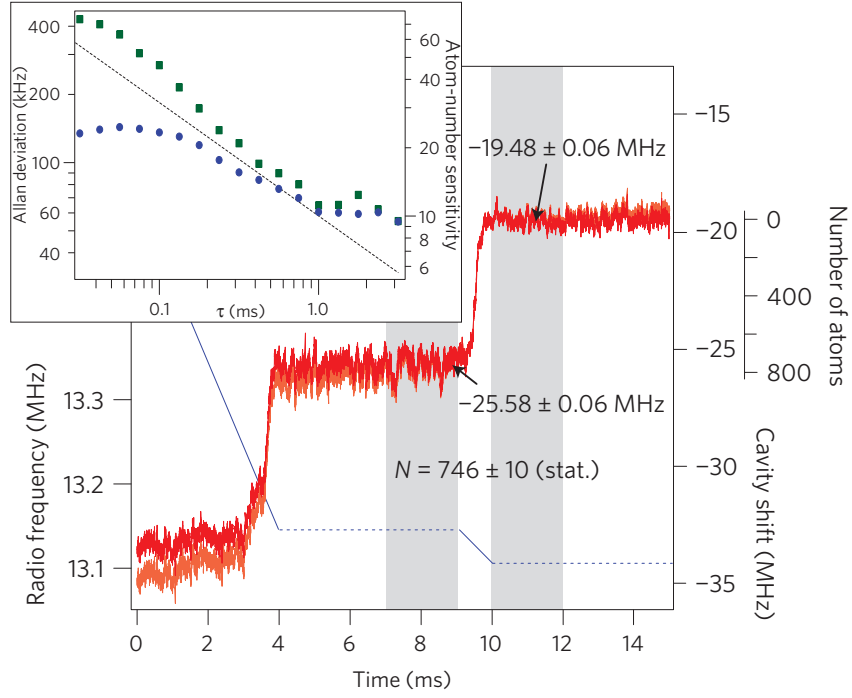

Figure 3 | Single-shot measurement of number of atoms in a single lattice site. The RF sweep (blue, left axis, solid line at full amplitude, dashed line at zero amplitude) is halted for a measurement period (shaded area), then swept across the lattice site of interest, before halting and measuring again. The number of atoms is calculated from the cavity shift (orange, right axis) after correcting for deterministic atom loss (red, right axis, corrected about $t=9.5 \mathrm{~ms}$ ). Here $\Delta_{\mathrm{ca}} / 2 \pi=-14 \mathrm{GHz}$, corresponding to 122 atoms $\mathrm{MHz}^{-1}$ of cavity shift. The inset shows the Allan deviation of the cavity shift after correcting for atom loss, using the probe frequency only (blue circles) and corrected using the instantaneous cavity transmission (green squares). The dotted line is the expected deviation due to photon shot noise. The

deviation on the probe frequency measurement at short times is below the shot-noise expectation due to a $20 \mathrm{kHz}$ electronic filter. The $60 \mathrm{kHz}$ Allan deviation for $\tau=2.2 \mathrm{~ms}$ corresponds to a sensitivity of ten atoms.

result of radiation pressure fluctuations caused by the probe light ${ }^{20}$, which reduce the trapping lifetime to $120 \mathrm{~ms}$, and spin decoherence and gradient-induced loss associated with executing the spin flips. Together, these processes result in $85 \%$ of the magnetization being preserved during the image. Less destructive imaging could be achieved at the expense of reduced signal-to-noise ratio or spatial resolution, for example by decreasing $\bar{\gamma}$, increasing the RF drive strength, or decreasing $B^{\prime}$.

Precise measurements of the longitudinal spin in a single lattice site can be taken by measuring the cavity resonance frequency before and after the spins in a single site are inverted (Fig. 3). The resonance frequency is first measured for a time $\tau$ with the RF off. The RF is then adiabatically turned on ( $125 \mu$ s linear ramp-on) at a frequency $20 \mathrm{kHz}$ above the spin-resonance frequency, chirped to $20 \mathrm{kHz}$ below the spin-resonance frequency, and adiabatically ramped off. Finally, $\Delta_{N}$ is again measured, and the total projection $S_{k}$ of the site's dimensionless spin is calculated using $S_{k}=\Delta \omega_{\mathrm{c}} / 2 C \bar{g}$, where $\Delta \omega_{\mathrm{c}}$ is the change in the cavity resonance frequency.

For small $\tau$, the precision of the single-site measurement is limited by the photon shot noise on the cavity resonance measurement to $\delta \omega_{\mathrm{c}}=\left|\mathrm{d} \omega_{\mathrm{c}} / \mathrm{d} \gamma\right| \times \sqrt{\bar{\gamma} / \varepsilon \tau}$, where $\varepsilon$ is the photodetection quantum efficiency and $\left|\mathrm{d} \omega_{\mathrm{c}} / \mathrm{d} \gamma\right|$ is the resonance frequency measurement sensitivity, $\simeq 11$ for our system (see Supplementary Information). For $\tau$ above $1 \mathrm{~ms}$, a measurement of the Allan deviation (Fig. 3) of $\omega_{\mathrm{c}}$ indicates that photon shot noise is superseded by technical noise, for example variations of the laser frequency or of $\omega_{0}$, yielding a frequency uncertainty of $60 \mathrm{kHz}$ for $\tau=2.2 \mathrm{~ms}$. With $\Delta_{\mathrm{ca}}=-14 \mathrm{GHz}$, the number of atoms in a single lattice site is thus determined with an rms uncertainty $\delta N=10$. This sensitivity should be sufficient to observe atom-number differences between lattice sites ${ }^{25}$ below the limit of Poissonian statistics $(\delta N \sim 30$ for $N \sim 1,000)$, while maintaining the probed gas for further experiments. We have also measured populations to $\delta N=2.5$, by using $\Delta_{\mathrm{ca}}=-2.0 \mathrm{GHz}$, although at this detuning the dynamic range of our measurement is limited to $N \lesssim 70$ within each site. Systematic uncertainties (Supplementary Information) affect the accuracy of the single-site measurement on the few-per cent level but do not significantly impact the measurement precision.

We use MRI to probe the transport dynamics of an atomic gas in an optical lattice under the effects of atomic interactions. We begin with atoms localized to a few lattice sites and image the initial stages of their expansion. We prepare a nondegenerate spin-polarized sample at $380 \mathrm{nK}$, with an initial width of $380 \mathrm{~nm}$. We allow the gas to evolve for variable time $t$ in a shallow optical lattice, which has a potential depth of $10.1 E_{r}$, where $E_{r}=h \times 3.17 \mathrm{kHz}$ is the rubidium recoil energy at a wavelength of $850 \mathrm{~nm}$. The lattice depth is then raised to perform MRI. The gravitational force is compensated by applying a weak levitating magnetic field gradient $\left(\mu B^{\prime} / h=454 \mathrm{~Hz}\right.$ per lattice constant) during transport. The experiment is repeated 15 times at each of several different values of $t$, and the images for each hold time are averaged together, after correcting for shot-to-shot variations of the bias field (Fig. 4).

The position variance of the atomic distribution grows as a result of quantum tunnelling. At early times $(t \leq 4 \mathrm{~ms})$ the growth matches the ballistic expansion of non-interacting atoms
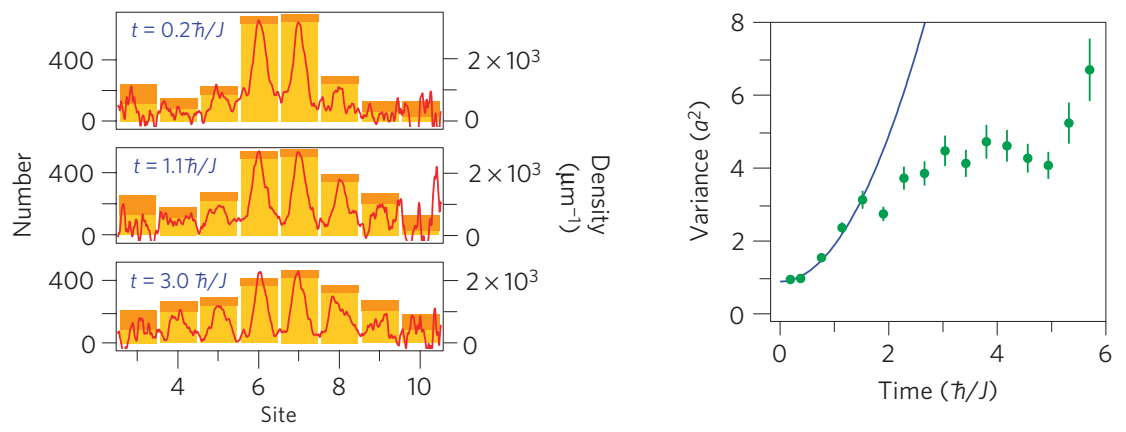

Figure 4 | Atom transport in an optical lattice as measured using cavity-aided MRI. ${ }^{87} \mathrm{Rb}$ atoms at $380 \mathrm{nK}$ tunnel resonantly in a $10.1 E_{r}$ lattice with tunnelling matrix element $J=\hbar \times 380 \mathrm{~s}^{-1}$. Left: after allowing the atoms to evolve for a fixed time $(t=0.2,1.1,3.0 \hbar / J$ shown), we use MRI to image the gas, correcting for spatially varying sensitivity. For each evolution time, 15 images are averaged together (red line, right axis), and these are integrated to obtain the atom-number distribution among lattice sites (yellow bars, left axis, orange region indicates 68\% certainty as obtained from Allan deviation). Each distribution is then fitted to a Gaussian envelope. Right: position variance $\sigma^{2}$ of the Gaussian envelope fit, expressed in units of square lattice spacing, as a function of transport time, expressed in units of inverse tunnelling rate. At early times $(t<2 \hbar / J)$, the data (green circles, error bars denote $68 \%$ certainty from fits) agree with no-free-parameter ballistic tunnelling theory (blue line). 
in the lattice, for an initial atomic axial distribution given by an incoherent mixture of single-site Wannier states. At later times the expansion slows markedly. Previous experiments have observed self-trapping in Bose-Einstein condensates ${ }^{26}$. The behaviour we observe agrees qualitatively with our simulations of interactioninduced self-trapping in a nondegenerate gas, enabled in this experiment by high atom density.

Using dispersive optical measurement, cavity enhancement, and magnetic resonance, we have demonstrated a spin-sensitive technique for imaging atomic gases with a spatial resolution of $150 \mathrm{~nm}$. The single-shot atom-number sensitivity is as small as 2.5 within a single lattice site, low enough to observe sub-Poissonian statistics of atom-number differences between sites with more than 10 atoms. Extending this technique to single-spin and single-atom counting should be possible by reducing technical measurement noise and increasing the experimental photon detection efficiency, currently limited by cavity losses.

The ability to control and to read out spins in single lattice sites provides new tools to engineer and study atomic gases at a microscopic level. Here we have measured the initial dynamics of bosonic atom transport in a 1D lattice with single-site resolution. Other experiments could include the observation of cavity-mediated long-range interactions between independently prepared spin populations or the observation of individual magnetic domains for antiferromagnetically ordered systems. Finally, because our dispersive measurement can be made minimally destructive, the method could be used to provide real-time feedback to spin projections of individual lattice sites for studies of quantum measurement and control $^{27}$, and for applications to atomic magnetometry.

\section{Received 9 December 2010; accepted 7 March 2011;} published online 10 April 2011

\section{References}

1. Bakr, W. S. et al. A quantum gas microscope for detecting single atoms in a Hubbard-regime optical lattice. Nature 462, 74-77 (2009).

2. Sherson, J. F. et al. Single-atom-resolved fluorescence imaging of an atomic Mott insulator. Nature 467, 68-72 (2010).

3. Schrader, D. et al. Neutral atom quantum register. Phys. Rev. Lett. 93, 150501 (2004).

4. Vengalattore, M. et al. High-resolution magnetometry with a spinor Bose-Einstein condensate. Phys. Rev. Lett. 98, 200801 (2007).

5. Shin, Y. I., Schunck, C. H., Schirotzek, A. \& Ketterle, W. Phase diagram of a two-component Fermi gas with resonant interactions. Nature 451, 689-693 (2008).

6. Ho, T. L. \& Zhou, Q. Obtaining the phase diagram and thermodynamic quantities of bulk systems from the densities of trapped gases. Nature Phys. 6, 66-69 (2010).

7. Navon, N., Nascimbene, S., Chevy, F. \& Salomon, C. The equation of state of a low-temperature Fermi gas with tunable interactions. Science 328, 729-732 (2010).

8. Horikoshi, M., Nakajima, S., Ueda, M. \& Mukaiyama, T. Measurement of universal thermodynamic functions for a unitary Fermi gas. Science 327, 442-445 (2010).
9. Weitenberg, C. et al. Single-spin addressing in an atomic Mott insulator. Nature 471, 319-324 (2011).

10. Würtz, P., Langen, T., Gericke, T., Koglbauer, A. \& Ott, H. Experimental demonstration of single-site addressability in a two-dimensional optical lattice. Phys. Rev. Lett. 103, 080404 (2009).

11. Scheunemann, R., Cataliotti, F. S., Hansch, T. W. \& Weitz, M. Resolving and addressing atoms in individual sites of a $\mathrm{CO}_{2}$-laser optical lattice. Phys. Rev. A 62, 051801 (2000).

12. Nelson, K. D., Li, X. \& Weiss, D. S. Imaging single atoms in a three-dimensional array. Nature Phys. 3, 556-560 (2007).

13. Karski, M. et al. Nearest-neighbour detection of atoms in a 1D optical lattice by fluorescence imaging. Phys. Rev. Lett. 102, 053001 (2009).

14. Gericke, T. et al. High-resolution scanning electron microscopy of an ultracold quantum gas. Nature Phys. 4, 949-953 (2008).

15. Khudaverdyan, M. et al. Quantum jumps and spin dynamics of interacting atoms in a strongly coupled atom-cavity system. Phys. Rev. Lett. 103, 123006 (2009).

16. Gehr, R. et al. Cavity-based single atom preparation and high-fidelity hyperfine state readout. Phys. Rev. Lett. 104, 203602 (2010).

17. Leroux, I. D., Schleier-Smith, M. H. \& Vuletić, V. Implementation of cavity squeezing of a collective atomic spin. Phys. Rev. Lett. 104, 073602 (2010).

18. Karski, M. et al. Imprinting patterns of neutral atoms in an optical lattice using magnetic resonance techniques. New J. Phys. 12, 065027 (2010).

19. Brahms, N. \& Stamper-Kurn, D. M. Spin optodynamics analog of cavity optomechanics. Phys. Rev. A 82, 041804 (2010).

20. Murch, K. W., Moore, K. L., Gupta, S. \& Stamper-Kurn, D. M. Observation of quantum-measurement backaction with an ultracold atomic gas. Nature Phys. 4, 561-564 (2008).

21. Brennecke, F., Ritter, S., Donner, T. \& Esslinger, T. Cavity optomechanics with a Bose-Einstein condensate. Science 322, 235-238 (2008).

22. Purdy, T. et al. Tunable optomechanics with ultracold atoms. Phys. Rev. Lett. 105, 133701 (2010).

23. Kuzmich, A. et al. Quantum nondemolition measurements of collective atomic spin. Phys. Rev. A 60, 2346-2350 (1999).

24. Rowland, S. W. in Image Reconstruction from Projections 1 st edn (ed. Herman, G. T.) (Springer, 1979).

25. Esteve, J. et al. Squeezing and entanglement in a Bose-Einstein condensate. Nature 455, 1216-1219 (2008).

26. Anker, T. et al. Nonlinear self-trapping of matter waves in periodic potentials Phys. Rev. Lett. 94, 020403 (2005).

27. Förster, L. et al. Microwave control of atomic motion in optical lattices. Phys. Rev. Lett. 103, 233001 (2009).

\section{Acknowledgements}

This work was supported by NSF Grant \#PHY-0801827 and the AFOSR. D.M.S-K. acknowledges support from the Miller Institute for Basic Science, and T.B. acknowledges support from Le Fonds Québécois de la Recherche sur la Nature et les Technologies. The authors thank A. M. Rey and K. Hazzard for discussions on the transport experiment.

\section{Author contributions}

Experimental data were taken by T.P.P., N.B., D.W.C.B. and T.B. All authors were involved with experimental design, data analysis, and production of the manuscript.

\section{Additional information}

The authors declare no competing financial interests. Supplementary information accompanies this paper on www.nature.com/naturephysics. Reprints and permissions information is available online at http://npg.nature.com/reprintsandpermissions. Correspondence and requests for materials should be addressed to N.B. 